\title{
Multiple bilateral malar mucinous cystadenomas in the minor salivary glands
}

Jun Ho Choi,

Seung Hyun Kim,

Jae Ha Hwang,

Kwang Seog Kim, Sam Yong Lee

Department of Plastic and

Reconstructive Surgery, Chonnam

National University Medical School,

Gwangju, Korea

\begin{abstract}
Mucinous cystadenoma, one of the subtypes of cystadenomas, is a rare benign salivary gland tumor. Most of the cases reported tumors presenting as asymptomatic, slow growing, single masses, primarily occurring in the parotid glands, buccal mucosa, and hard palate. This report describes a case of multiple mucinous cystadenomas that presented as subcutaneous swellings in both cheeks, which were mistaken for a benign subcutaneous tumor. A complete surgical excision was performed through an intraoral incision. There were no recurrences or complications. A diagnosis of mucinous cystadenoma was made by histopathological examination. Mucinous cystadenomas are differentiated from other salivary gland tumors based on the pathological findings. A malignant transformation is also possible. Preoperative imaging cannot identify mucinous cystadenomas because of their small size and atypical features, and needle biopsy has its limitations in such salivary gland tumors. Therefore, accurate diagnosis and treatment through surgical excision become important.
\end{abstract}

Keywords: Cheek / Minor salivary gland / Mucinous cystadenoma

\section{INTRODUCTION}

Mucinous cystadenoma is a benign cystic tumor lined with mucinous epithelium. These mucinous cystadenoma subtypes are known to occur in the ovaries, pancreas, peritoneum, and liver. In general, mucinous cystadenomas at these different locations are considered to exist independently [1-3]. Salivary gland tumors account for $3 \%$ of head and neck tumors, most of which occur in the parotid gland, and less than $25 \%$ of which are minor salivary gland tumors [4].

Cystadenoma of the salivary glands is a rare benign epithelial tumor, accounting for $4.2 \%-4.7 \%$ of all benign salivary gland tumors. Distribution sites include the parotid gland (58\%), submandibular gland $(6.6 \%)$, sublingual gland $(0.5 \%)$, minor salivary glands in the lips (10.7\%), and palate (7.1\%) [5]. Accord-

\section{Correspondence: Jae Ha Hwang}

Department of Plastic and Reconstructive Surgery, Chonnam National University

Medical School, 42 Jebong-ro, Dong-gu, Gwangju 61469, Korea

E-mail: psjhhwang@daum.net

Received July 20, 2020 / Revised August 18, 2020 / Accepted October 15, 2020 ing to the World Health Organization's Histological Classification of Salivary Gland Tumors, these are divided into papillary and mucinous subtypes, the latter being uncommon. In general, this type is predominant among women, and $45 \%$ of the reported cases involved the parotid gland and minor salivary glands in the lip and buccal mucosa. Clinically, the tumor presents as a slow growing painless mass in the major salivary glands, and as a smooth-surfaced nodule, similar to a mucocele, in the oral mucosa [6,7].

In particular, the mucinous type of cystadenomas is relatively rare. The few cases that have been reported were in the parotid gland, buccal mucosa, and palate. In Korea, two cases involving the parotid glands have been reported [8-10].

In this paper, we report a case of multiple mucinous cystadenomas in an atypical site, presenting as subcutaneous nodules in both cheeks. In this case, excisional biopsy was conducted because the nodules distributed in the subcutaneous layer of both cheeks were not distinguishable from a benign subcutaneous tumor, even after ultrasonography and computed tomogra- 
phy (CT). Therefore, the patient was diagnosed with mucinous cystadenoma occurring in an atypical region.

\section{CASE REPORT}

A 37-year-old woman presented with multiple nodules over both the nasolabial folds. These had been first noticed 3 months
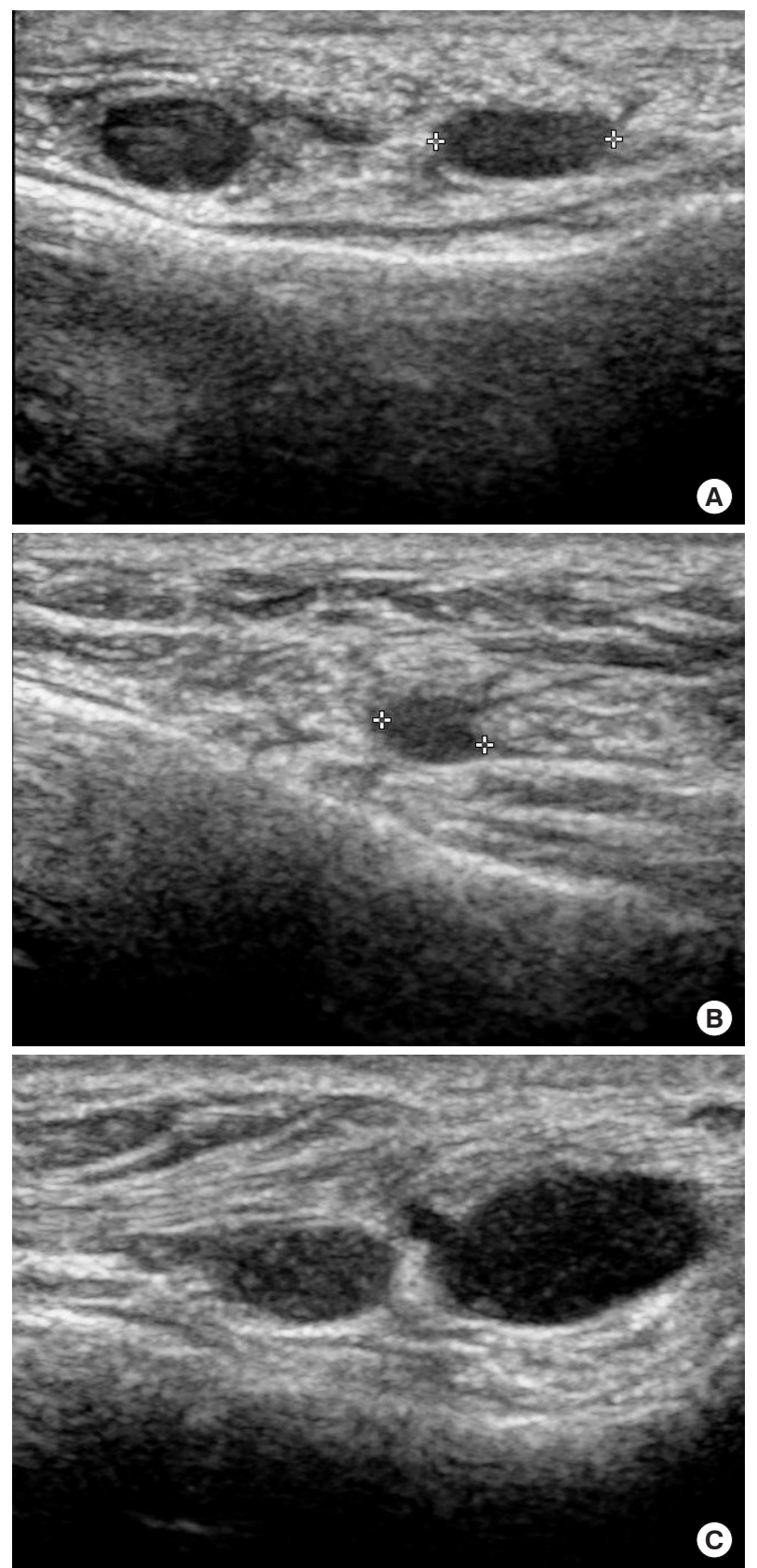

Fig. 1. Preoperative ultrasonography. (A) Two $0.5 \mathrm{~cm}$-sized masses seen in the subcutaneous layer of the left cheek. (B) A mass measuring $0.3 \mathrm{~cm}$ in the left cheek. (C) Two subcutaneous masses measuring 0.5 and $0.8 \mathrm{~cm}$ in the right cheek. According to ultrasonography reports, well-defined oval hypoechoic lesions in the subcutaneous layer were observed, possibly representing benign tumors. prior. Initially, two nodules occurred on the right side, followed by three on the left. They gradually increased in size, and the ones on the right appeared to spread to the left. On palpation, the nodules were very soft and non-tender. She had undergone augmentation rhinoplasty using silicone implants 10 years prior and intense pulsed light laser therapy for bilateral cheek melasma thrice in 2006.

Ultrasonography revealed well-defined oval hypoechoic lesions in the subcutaneous layer, with two lesions measuring 0.8 $\mathrm{cm}$ and $0.5 \mathrm{~cm}$ in the right cheek, and three lesions measuring $0.5 \mathrm{~cm}, 0.5 \mathrm{~cm}$, and $0.3 \mathrm{~cm}$ in the left cheek, which were predicted to be benign (Fig. 1). A contrast-enhanced CT of the face was performed for precise assessment and to rule out the possibility of any additional tumors. However, as there were no enhancing masses or enlarged lymph nodes, excisional biopsies targeting all masses were performed through the upper gingivobuccal sulcus under local anesthesia (Fig. 2). Histopathological assessment revealed epithelial cells lining a cystic cavity with mucous secretions, leading to the diagnosis of a benign mucinous cystadenoma (Fig. 3). The patient recovered well and her postopera-

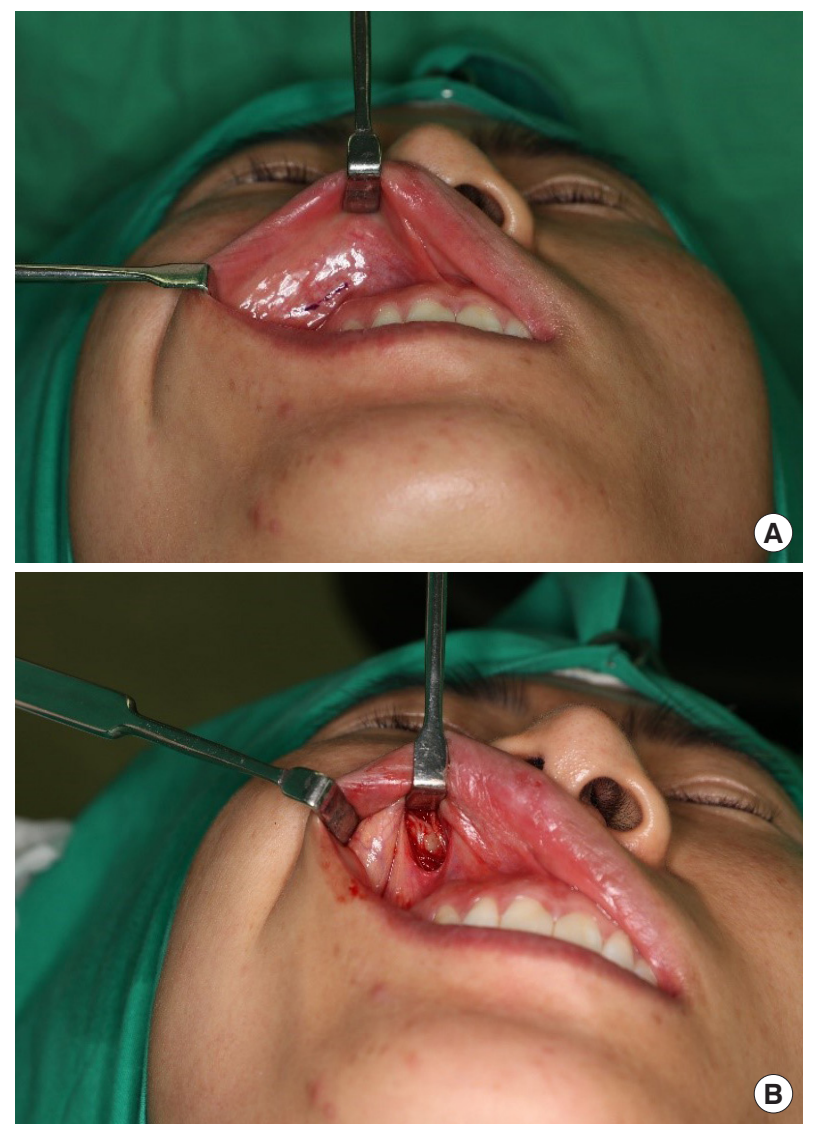

Fig. 2. Intraoperative photographs. (A) The masses were approached through an incision in the upper gingivobuccal sulcus. (B) Yellowish nodular masses were identified in the subcutaneous layer and complete excision was performed with primary closure. 

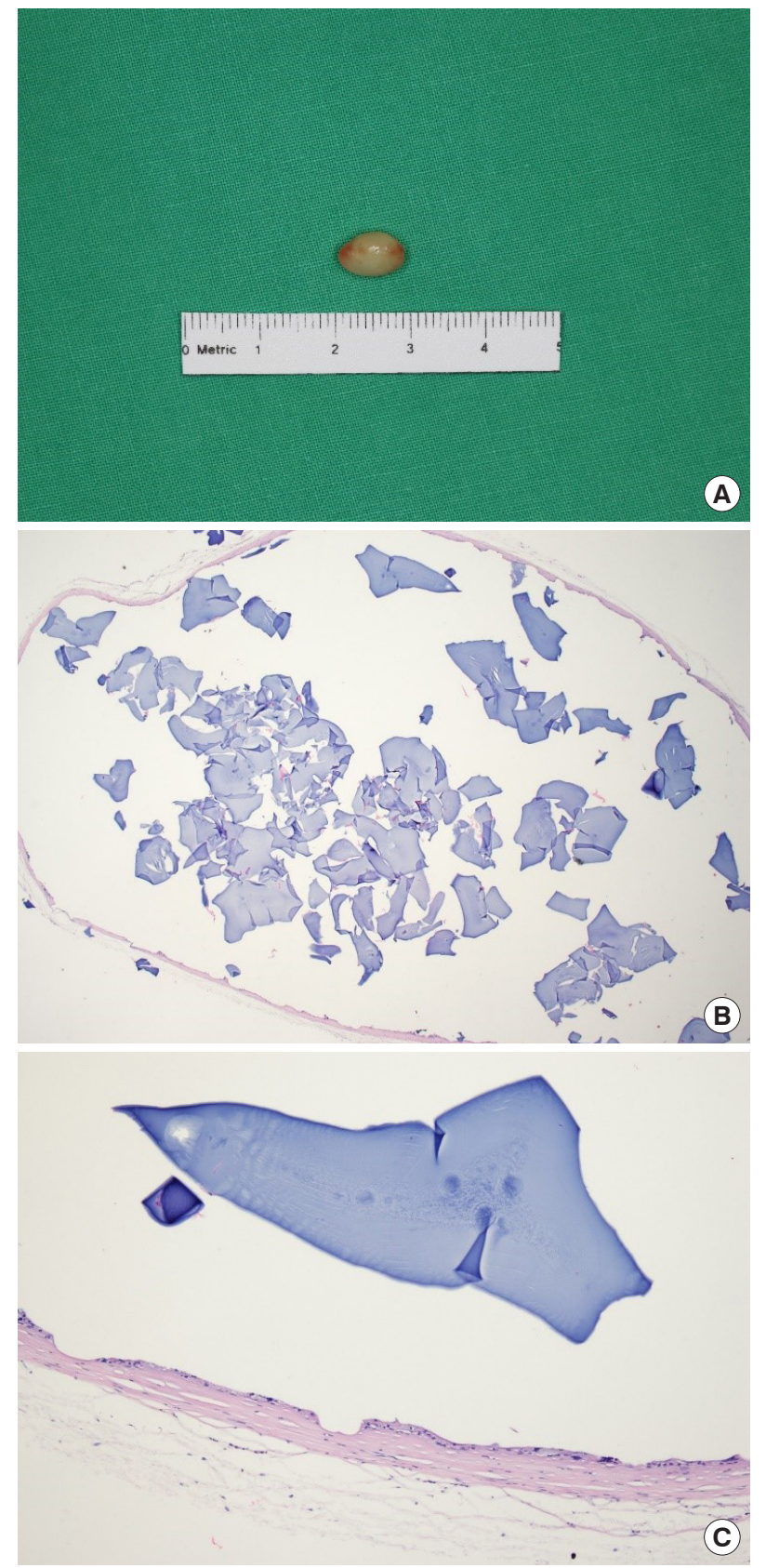

Fig. 3. Gross and histopathological images of the tumors. (A) Gross image of the $0.8 \mathrm{~cm}$ wide whole mass, which was one of the largest ones. (B) Histopathological image $(\mathrm{H} \& \mathrm{E}, \times 40)$. (C) Histopathological assessment revealed a layer of epithelial cells lining a cystic cavity with mucous secretions $(\mathrm{H} \& \mathrm{E}, \times 100)$.

tive period was uneventful. Follow-up at 6 months revealed no recurrence and excellent cosmetic results without scars.

\section{DISCUSSION}

Mucinous cystadenoma is defined as a cyst lined by mucous tall columnar epithelium with limited papillary growth. In the global literature, single cases of occurrence in the parotid gland, buccal mucosa, and hard palate have been reported. In Korea, only two cases of mucinous cystadenoma of the parotid gland have been published. In these reports, clinical features revealed a slow growing and asymptomatic mass similar to cystadenoma. In the case of a single mass in the major salivary glands, the diameter was $2.6 \mathrm{~cm}$, while that of the mass in the minor salivary glands was $2.5 \mathrm{~cm}[8-10]$.

In our case, five well-encapsulated subcutaneous masses, which were slow growing and painless, were found bilaterally in the cheeks. The largest mass measured only $0.8 \mathrm{~cm}$, and was not even detected on CT imaging. Additional salivary gland abnormalities were not observed. Ultrasonographical assessment confirmed that the lesions were located in the subcutaneous layer. Therefore, considering the atypical location and the presence of multiple lesions, our clinical diagnosis was inaccurate. In light of a suspected benign subcutaneous tumor, surgery was performed.

Fine-needle aspiration biopsy is generally performed as a diagnostic tool for salivary gland tumors before planning surgery. In this patient, the detected masses were small and slow growing, and the margins were regular with no vascularity observed on imaging. Hence, it was assumed to be benign or a low-grade malignancy and surgery was performed accordingly. It is important to mention that a case of mucinous cystadenoma in the parotid gland has been reported, where an ultrasonography guided needle biopsy was performed, without yielding a diagnosis [9]. This reveals that, in mucinous cystadenoma or a cyst surrounded by epithelium with thick fibrous tissue, a needle biopsy does not yield much information; thus, a needle biopsy may not help distinguish it from other salivary gland tumors.

The occurrence of mucinous cystadenoma in the subcutaneous layer of the cheek can be explained by the wide distribution of the minor salivary glands. They are present in the buccal mucosa, labial mucosa, palate, and submucosa of the aerodigestive tract, including the sinonasal cavity, oral cavity, pharynx, lungs, and middle ear cavity [11,12]. A minor salivary gland tumor in the submucosal layer of the upper gingivobuccal sulcus can present as a subcutaneous swelling in the malar region, which in our case led to the misdiagnosis of a benign soft tissue tumor.

Mucinous cystadenomas need to be differentiated from papillary cystadenomas, intraductal papillomas, cyst adenocarcinomas, low-grade mucoepidermoid carcinomas, Warthin's tumors, and polycystic diseases. This distinction requires studying the different histopathological aspects, such as the destruction of papillary projections and glandular parenchyma, invasion of surrounding tissues, stromal lymphoid aggregation of the germinal center, and diffuse polycystic changes of the entire gland [6]. 
In addition, there have been cases of malignant changes in mucinous cystadenoma; one of the studies reported a malignant transformation of the mucinous cystadenoma in the parotid glands into an invasive micropapillary adenocarcinoma [13]. So far, no reports on malignant transformations of minor salivary gland cystadenoma have been published.

Generally, benign cystadenoma and salivary gland malignancies, such as cystadenocarcinoma and low-grade mucoepidermoid carcinoma that may be mistaken for benign cystadenoma, are clinically similar. Thus, it becomes difficult to discriminate them only by physical examination. However, biopsies reveal pathological features linked to malignancy, such as the invasion of adjacent tissue, perineural or lymphatic/vascular invasion, increased mitotic activity, and cytologic pleomorphism, which facilitates differentiation $[7,14]$.

The prognosis of this tumor is good and the likelihood of recurrence is low. Incomplete removal could lead to recurrence, and reports of recurrence have mostly involved the major rather than the minor salivary glands [15].

This case is an example of multiple mucinous cystadenomas that presented as tumors in the subcutaneous layer of both cheeks. Due to their atypical features and location, a preoperative diagnosis was difficult here despite conducting imaging studies. However, a complete surgical excision via an intraoral approach resulted in an accurate diagnosis and a scarless outcome.

This case demonstrates that rare salivary gland tumors in atypical locations can be challenging to diagnose. In case of mucinous cystadenomas, it is recommended to reach an accurate diagnosis histopathologically and perform complete excision to prevent recurrence and malignant transformation.

\section{NOTES}

\section{Conflict of interest}

No potential conflict of interest relevant to this article was reported.

\section{Ethical approval}

The study was approved by the Institutional Review Board of Chonnam National University Hospital (IRB No. CNUHEXP-2020-205) and performed in accordance with the principles of the Declaration of Helsinki. Written informed consent was obtained.

\section{Patient consent}

The patient provided written informed consent for the publication and the use of her images.

\section{ORCID}

Jun Ho Choi

Seung Hyun Kim

Jae Ha Hwang

Kwang Seog Kim

Sam Yong Lee

\section{REFERENCES}

1. Hart WR. Mucinous tumors of the ovary: a review. Int J Gynecol Pathol 2005;24:4-25.

2. Weledji EP, Eyongetah D, Nana TC, Ngowe MN. Excision of mucinous cystadenoma of pancreas is safe and effective: a case report. Int J Surg Oncol 2018;3:e47.

3. Lee SY, Han WC. Primary retroperitoneal mucinous cystadenoma. Ann Coloproctol 2016;32:33-7.

4. Auclair PL, Ellis GL, Gnepp DR, Wenig BM, Janney CG. Salivary gland neoplasms: general considerations. In: Ellis GL, Auclair PL, Gnepp DR, editors. Surgical pathology of the salivary glands. Philadelphia: W.B. Saunders Co.; 1991. p. 135-64.

5. Goto M, Ohnishi Y, Shoju Y, Wato M, Kakudo K. Papillary oncocytic cystadenoma of a palatal minor salivary gland: a case report. Oncol Lett 2016;11:1220-2.

6. Skalova A, Michal M. Cystadenoma. In: Barnes L, Eveson JW, Reichart D, editors. World Health Organization classification of tumours pathology and genetics of head and neck tumours. Lyon: IARC Press; 2005. p. 273-4.

7. Seethala RR, Stenman G. Update from the 4 th edition of the World Health Organization classification of head and neck tumours: tumors of the salivary gland. Head Neck Pathol 2017; 11:55-67.

8. Cho MJ, Kang YJ, Choi DJ, Lee CK. A case of mucinous cystadenoma of the parotid gland. J Korean Soc Plast Reconstr Surg 1995;22:196-200.

9. Ryou N, Ko DY, Jung H, Cho JG. A case of mucinous cystadenoma mimicking mucoepidermoid carcinoma in parotid gland. Korean J Otorhinolaryngol-Head Neck Surg 2015;58: 886-9.

10. Foss RD, Ellis GL, Auclair PL. Salivary gland cystadenocarcinomas: a clinicopathologic study of 57 cases. Am J Surg Pathol 1996;20:1440-7.

11. Kessler AT, Bhatt AA. Review of the major and minor salivary glands, part 1: anatomy, infectious, and inflammatory processes. J Clin Imaging Sci 2018;8:47.

12. Kim YH, Yoon HW, Kim J, Kim SW. Ectopic pleomorphic adenoma on subcutaneous plane of the cheek. Arch Craniofac Surg 2019;20:55-7.

13. Michal M, Skalova A, Mukensnabl P. Micropapillary carcino- 
ma of the parotid gland arising in mucinous cystadenoma. Virchows Arch 2000;437:465-8.

14. Oh SJ, Moon D. Spindle cell myoepithelioma of the parotid gland. Arch Craniofac Surg 2019;20:336-40.
15. Tjioe KC, de Lima HG, Thompson LD, Lara VS, Damante JH, de Oliveira-Santos C. Papillary cystadenoma of minor salivary glands: report of 11 cases and review of the English literature. Head Neck Pathol 2015;9:354-9. 